\title{
Seleção de variáveis em modelos matemáticos dos parâmetros de cultivo do camarão marinho Litopenaeus vannamei
}

\author{
Ady Marinho Bezerra(1), José Antônio Aleixo da Silva(1) e Paulo de Paula Mendes ${ }^{(1)}$ \\ (1)Universidade Federal Rural de Pernambuco, Rua D. Manoel de Medeiros, s/no, Dois Irmãos, CEP 52171-030 Recife, PE. \\ E-mail: amarinho@jc.com.br, jaaleixo@uol.com.br, paulo_ufrpe@yahoo.com.br
}

\begin{abstract}
Resumo - O objetivo deste trabalho foi selecionar as variáveis de manejo do camarão marinho Litopenaeus vannamei que mais influenciaram nas variáveis-respostas ao cultivo (produção, produtividade, peso final e taxa de sobrevivência), em modelos matemáticos. O banco de dados foi composto por 83 cultivos, realizados no período de 2003 a 2005, obtidos de uma fazenda comercial localizada no litoral sul de Pernambuco. Para estimar os parâmetros dos modelos, utilizou-se a técnica dos mínimos quadrados. A seleção das variáveis foi realizada com o processo "backward elimination" associado ao método de transformação de Box e Cox. A adequação das equações e os pressupostos de normalidade e homocedasticidade, para os erros, foram analisadas com base na análise de variância e análise de resíduo. É possível relacionar essas variáveis e estabelecer predições com as equações.
\end{abstract}

Termos para indexação: criação, regressão, Box, Cox, stepwise.

\section{Selection of variables in mathematical models of culture parameters of marine shrimp Litopenaeus vannamei}

\begin{abstract}
The objective of this work was to select management variables of the marine shrimp Litopenaeus vannamei that most influenced culture variable responses (production, productivity, final weight and survival rate), in mathematical models. The database was composed of 83 cultures in the period of 2003 to 2005, obtained from a shrimp farm located in the South coast of Pernambuco. To estimate the parameters of the models it was used the technique of least square. The selection of variable was carried through the backward elimination process associated to the Box and Cox transformation. The adequacy of the equations and the hypothesis of normality and homogeneous variance for the errors were analyzed based on the analysis of variance and on the analysis of residuals. It is possible to correlate those variables and to establish predictions with the equations.
\end{abstract}

Index terms: rearing, regression, Box, Cox, stepwise.

\section{Introdução}

A produção mundial de camarões cultivados e capturados, em 2004, foi da ordem de 5,328 milhões de toneladas, das quais $33,87 \%$ vieram dos cultivos (FAO, 2006). Entre os fatores que contribuíram para esta elevada produção, destacam-se as pesquisas na área tecnológica, a elevada demanda do mercado mundial do setor e a redução na produção de camarão oriundo da pesca extrativa. O Brasil obteve, nesses últimos anos, a liderança mundial em produtividade e, em 2003, a maior produção do camarão Litopenaeus vannamei do hemisfério ocidental. Nesse mesmo ano, verificou-se a primeira queda na produção e produtividade brasileira dos últimos sete anos, que foi de 15,8 e $25,9 \%$, respectivamente, em relação ao ano anterior. Essa queda decorreu, principalmente, da incidência do vírus IMNV (Mionecrose Infecciosa), da baixa cotação do dólar e o "dumping" promovido pelos Estados Unidos contra o produto brasileiro (Rodrigues, 2005).

As espécies de camarão mais cultivadas no mundo são Litopenaeus vannamei $(40,66 \%)$, Penaeus monodon $(37,41 \%)$ e Fenneropenaeus chinensis (10,97\%) (FAO, 2006). O Litopenaeus vannamei é, praticamente, a única espécie utilizada em cultivo comercial de camarão marinho no Brasil (Nunes, 2004) e essa espécie, em água doce, tem apresentado bom crescimento (Boyd, 1997). 
De acordo com o censo de 2004, o número de produtores de camarão no Brasil é de 997, dos quais 98 $(9,83 \%)$ deles estão em Pernambuco. Em geral, as fazendas de camarão brasileiras adotam o sistema bifásico de cultivo, ou seja, a fase berçário e a fase de engorda (crescimento). Na fase berçário, as pós-larvas (PL) são colocadas em tanques-berçário, por um período médio de 12 dias, local em que elas são aclimatadas às futuras condições de campo; na fase de engorda, as pós-larvas são transferidas dos tanques-berçário para os viveiros de engorda, e os camarões são estocados em densidades que variam de 20 a 80 camarões por metro quadrado, durante 100 a 120 dias.

Além das técnicas de manejo, utilizadas nos cultivos dos camarões, recentemente tem-se destacado o uso da estatística na análise do banco de dados dessas fazendas, para modelar os parâmetros relacionados ao cultivo e, assim, melhorar a produção e diminuir os custos. Entre essas técnicas, destacam-se as aplicações das regressões lineares, em especial as múltiplas, com as quais se podem estimar os parâmetros das variáveisrespostas ao cultivo. Ao associá-las à técnica de seleção de variáveis, é possível detectar aquelas que mais influenciaram em cada resposta de produção (Pereira, 2001; Ximenes, 2005).

O objetivo deste trabalho foi selecionar as variáveis de manejo do camarão marinho Litopenaeus vannamei que mais influenciaram nas variáveis-respostas ao cultivo (produção, produtividade, peso final e taxa de sobrevivência), em modelos matemáticos.

\section{Material e Métodos}

Foi utilizado um banco de dados de uma fazenda comercial de cultivo do camarão marinho Litopenaeus vannamei, localizada no litoral sul de Pernambuco. O sistema de cultivo adotado pela fazenda é o bifásico, compreendido pelas fases de berçário e de engorda. Para a fase berçário, a fazenda disponibiliza seis tanques circulares, em alvenaria, cada um com capacidade de 50 mil litros. Os tanques-berçário possuem um sistema de aeração abastecido por três sopradores radiais (7,5 HP). Nessa fase, são estocadas 20 a 25 pós-larvas por litro; o tempo médio de cultivo é de 10 a 12 dias, e a dieta é à base de biomassa de artêmia e ração comercial administradas a cada 2 horas, durante o dia. Para a fase de engorda, dispõe-se de 12 viveiros, com áreas que variam de 3,24 ha a 5,45 ha, com profundidade média de $2 \mathrm{~m}$ em seu centro. No final de cada cultivo, o viveiro é submetido a um período médio de vazio de 10 a 15 dias. A densidade de estocagem média foi de 63 camarões por metro quadrado e foram utilizados aeradores de pás com potência de 2 HP. Diariamente, a cada 2 horas, foram monitorados os parâmetros físicos e químicos da água dos viveiros.

Para a geração do banco de dados, destinados às análises, utilizou-se o período de 2003 a 2005, durante o qual foram realizados 83 cultivos. As variáveis-respostas de produção foram: produção (Prod), produtividade (Prodt), taxa de sobrevivência (Sob) e peso final (PF). As variáveis de manejo (independentes) foram: quantidade de ração ofertada (QRO), estação do ano inverno $\left(\mathrm{EA}_{\mathrm{I}}\right)$, estação do ano verão $(\mathrm{EA} V)$, tempo de cultivo (TC), densidade de estocagem (DE), laboratório fornecedor de pós-larva (LFPL), área do viveiro (AV), número de ciclos (NC), população inicial (PI), aeração (Aera), tempo de preparação (TP), salinidade (SAL), temperatura de fundo $\left(\mathrm{Temp}_{\mathrm{F}}\right)$, temperatura de superfície $\left(\mathrm{Temp}_{\mathrm{S}}\right)$, oxigênio de fundo $\left(\mathrm{Oxi}_{\mathrm{F}}\right)$, oxigênio de superfície $\left(\mathrm{Oxi}_{\mathrm{S}}\right), \mathrm{pH}(\mathrm{pH})$, nível da água (NA) e transparência da água (Transp).

As variáveis independentes foram relacionadas com as variáveis de manejo de acordo com o seguinte modelo:

$$
\mathrm{Y}_{\mathrm{i}}=\beta_{0}+\sum_{\mathrm{k}=1}^{\mathrm{K}} \beta_{\mathrm{ki}} \mathrm{X}_{\mathrm{ki}}+\varepsilon_{\mathrm{i}}
$$

em que: $Y_{\mathrm{i}}$ são as variáveis respostas (Prod, Prodt, Sob e $\mathrm{PF}) ; \mathrm{X}_{\mathrm{i}}$ são as variáveis de manejo (QRO, EA $\mathrm{E}, \mathrm{EA}$, DC, DE, LFPL, AV, NC, PI, Aera, DP, SAL, Temp F $_{\text {, Temps, }}$, Oxi $i_{F}$, Oxi $_{S}, p H$, NA e Transp); $\beta_{\mathrm{i}}$ são os parâmetros do modelo; $\varepsilon_{\mathrm{i}}$ são os erros associados à cada observação $\mathrm{e}$ possuem distribuição normal com os parâmetros $\left(0, \sigma^{2}\right)$; $\mathrm{K}$ é a enésima variável independente $(\mathrm{k}=1,2,3, \ldots, \mathrm{K}) \mathrm{e}$ i é a enésima observação ( $\mathrm{i}=1,2,3, \ldots, \mathrm{I})$.

Para selecionar as variáveis independentes, que influenciaram significativamente na relação com as respostas, utilizou-se o processo de seleção de variáveis "backward elimination". No referido processo, utilizouse a estatística $\mathrm{F}$ da análise de variância (ANOVA) de entrada $\left(\mathrm{F}_{\text {enter }}\right)$ e de exclusão $\left(\mathrm{F}_{\text {remover }}\right)$ de variáveis, ajustada para o valor 4 . Os parâmetros $\beta_{0}, \beta_{1}, \ldots, \beta_{\mathrm{k}}$ foram estimados com base nas técnicas dos mínimos quadrados. Para o ajuste do melhor modelo e normalização da variável-resposta, foi usado o processo de transformação de Box e Cox, apresentado por Montgomery \& Peck 
(1982), o qual objetiva estimar o valor de $\lambda$ que minimiza a soma dos quadrados dos erros $\left(\mathrm{SQ}_{\text {Resíduo }}\right)$, definido pela seguinte família de transformadores:

$\mathrm{W}_{\mathrm{i}}=\frac{\mathrm{Y}_{\mathrm{i}}^{\lambda}-1}{\lambda \dot{\mathrm{Y}}^{\lambda-1}}, \forall \lambda \neq 0$ e $\mathrm{W}_{\mathrm{i}}=\dot{\mathrm{Y}} \operatorname{Ln}\left(\mathrm{Y}_{\mathrm{i}}\right), \forall \lambda=0$

em que: $W$ é o valor transformado; $Y$ é o valor observado; $\lambda$ é a família de transformadores; Y é a média geométrica do vetor-resposta original e i é a enésima observação $(i=1,2,3, \ldots, I)$.

As variáveis LFPL (laboratório fornecedor de póslarva) e estação do ano (EA), por serem qualitativas, foram inseridas no modelo como variáveis binárias (0 ou 1), conforme recomendado por Mendes (1999). Foi considerado inverno o período de março a agosto, e verão de setembro a fevereiro. $\mathrm{O}$ banco de dados foi composto por cinco laboratórios fornecedores de póslarvas, os quais foram nomeados de A, B, C, D e E, aleatoriamente, como estratégia de preservar suas razões sociais.

Para verificar se a equação obtida foi adequada aos dados, realizou-se a análise de variância (ANOVA) para a regressão. A soma dos quadrados dos resíduos da ANOVA e o índice determinístico $\left(\mathrm{R}^{2}\right)$ foram as estatísticas utilizadas para se verificar o quanto as variáveis independentes, inseridas na equação, explicaram a variabilidade da variável-resposta. Para verificar se foram respeitados os pressupostos de linearidade, homocedasticidade e normalidade, para os erros, realizou-se a análise gráfica de resíduos, de acordo com as definições de Cordeiro \& Neto (2004).

\section{Resultados e Discussão}

Dos 83 dados de cultivo 18,07\% não foram utilizados em decorrência de se encontrarem incompletos quanto a algumas informações de manejo, ou as variáveis físicas ou químicas, ou quando apresentaram valores considerados errados. A sumarização da estatística desses cultivos está apresentada na Tabela 1.

Ao relacionar as variáveis de produção, produtividade, peso final e taxa de sobrevivência, em relação às variáveis de manejo e às físicas e químicas da água, verificou-se que a ração ofertada e a temperatura no fundo dos viveiros foram selecionadas para todos os modelos. As variáveis dias de cultivo e população inicial foram incluídas em três dos quatro modelos, e a área do viveiro, tempo de preparação, densidade inicial e aeração foram incluídos em dois modelos (Tabela 2).

De acordo com os resultados da estatística $F$ da ANOVA, pode-se afirmar $(\mathrm{p}<0,05)$ que as equações encontradas podem ser aplicadas aos dados. Apesar das transformações utilizadas $(\lambda)$ para cada modelo, verificou-se, com base na análise de resíduo, que os pressupostos normalidade e homocedasticidade, para os erros, foram atendidos. Os pontos discrepantes detectados foram mantidos na matriz de dados, pois se constatou que não houve erro de mensuração nem de transcrição de dados, de acordo com recomendações de Sokal \& Rohlf (1995), e que também não excedeu ao limite de tolerância de 5\% proposto por Mendes (1999).

Tabela 1. Estatística descritiva dos dados de cultivo do Litopenaeus vannamei.

\begin{tabular}{|c|c|c|c|}
\hline Variável & Mínimo & Máximo & Média $^{(1)} \pm$ erro $^{(2)}$ \\
\hline Sobrevivência (\%) & 45,86 & 93,44 & $74,53 \pm 0,03$ \\
\hline Peso médio $(\mathrm{g})$ & 9,06 & 16,30 & $12,84 \pm 0,40$ \\
\hline Produção $(\mathrm{t})$ & 11,73 & 42,28 & $25,44 \pm 1,53$ \\
\hline Produtividade ( $\mathrm{kg} \mathrm{ha}^{-1}$ por ano) & 9,54 & 23,42 & $15,96 \pm 0,68$ \\
\hline Número de ciclos & 6 & 12 & $\overline{9}$ \\
\hline Área do viveiro (ha) & 3,24 & 5,45 & $4,28 \pm 0,16$ \\
\hline Tempo de preparação (dias) & 5 & 38 & $14 \pm 1,42$ \\
\hline População inicial (indivíduos) & 1.420 .000 & 4.230 .000 & $2.704 .335 \pm 153.267$ \\
\hline Densidade de estocagem (indivíduo por $\mathrm{m}^{2}$ ) & 41 & 79 & $63 \pm 2,38$ \\
\hline Tempo de cultivo (dias) & 93 & 170 & $121 \pm 3,08$ \\
\hline Ração ofertada (kg) & 23.408 & 76.742 & $41.441 \pm 2.807$ \\
\hline Aerador $\left(\mathrm{HP} \mathrm{ha}^{-1}\right)$ & 6,30 & 20,00 & $13,24 \pm 0,80$ \\
\hline Nível de água (cm) & 74,66 & 92,00 & $85,27 \pm 0,83$ \\
\hline Oxigênio de fundo $\left(\mathrm{mg} \mathrm{L}^{-1}\right)$ & 5,07 & 7,69 & $6,37 \pm 0,15$ \\
\hline Oxigênio de superfície $\left(\mathrm{mg} \mathrm{L}^{-1}\right)$ & 5,30 & 8,41 & $6,81 \pm 0,13$ \\
\hline $\mathrm{pH}$ & 6,10 & 8,92 & $7,75 \pm 0,09$ \\
\hline Salinidade $\left(\mathrm{g} \mathrm{L}^{-1}\right)$ & 23 & 39 & $32 \pm 0,91$ \\
\hline Temperatura de fundo $\left({ }^{\circ} \mathrm{C}\right)$ & 27,09 & 31,07 & $29,78 \pm 0,24$ \\
\hline Temperatura de superfície $\left({ }^{\circ} \mathrm{C}\right)$ & 27,11 & 32,48 & $29,98 \pm 0,26$ \\
\hline Transparência $(\mathrm{cm})$ & 38,13 & 68,48 & $48,56 \pm 1,48$ \\
\hline
\end{tabular}

${ }^{(1)}$ Média obtida de 68 dados. ${ }^{(2)}$ Erro $=\mathrm{t}_{\alpha / 2} \cdot \mathrm{S}_{\overline{\mathrm{x}}}$. 
De acordo com o processo de seleção, ao relacionar as 18 variáveis de manejo com a variável-resposta produção, verificou-se que apenas 7 variáveis foram selecionadas (Equação 1):

Prod $=\left(13,63-1,73 \mathrm{NC}+28,55 \mathrm{AV}+1,18 \mathrm{SAL}-2,68 \mathrm{Temp}_{\mathrm{F}}\right.$ $\left.-3,18 \times 10^{-5} \mathrm{PI}+2,02 \mathrm{DE}+0,001 \mathrm{QRO}\right)^{2}$, em que: Prod é a produção (t); NC é o número de ciclos; AV é a área do viveiro (ha); SAL é a salinidade $\left(\mathrm{g} \mathrm{L}^{-1}\right)$; Temp $\mathrm{F}_{\mathrm{F}}$ é a temperatura de fundo $\left({ }^{\circ} \mathrm{C}\right)$; PI é a população inicial; DE é a densidade de estocagem (camarão por $\mathrm{m}^{2}$ ); QRO é a quantidade de ração ofertada $(\mathrm{kg})$.

Com base na equação de produção (Equação 1), verificou-se que o valor do transformador de Box e Cox, que minimizou a $\mathrm{SQ}_{\mathrm{Resíduo}}$, ocorreu quando o vetorresposta Prod foi submetido ao transformador $\lambda=0,5$, ou seja, $\left(\operatorname{Prod}^{0,5}\right)$. Nessas condições, apenas as variáveis: $\mathrm{NC}, \mathrm{AV}, \mathrm{SAL}, \mathrm{Temp}_{\mathrm{F}}, \mathrm{PI}, \mathrm{DE}$ e QRO foram consideradas significativas para estimar Prod $(\mathrm{p}<0,0001)$. Ao se manter as variáveis AV, SAL, DE e QRO em seus valores máximos (Tabela 1), obtém-se a maximização da produção. No entanto, ao condicionar a $\mathrm{Temp}_{\mathrm{F}}$ em seu valor máximo, o número máximo de ciclos e estocando-se o máximo de indivíduos, estimouse a menor produção. De acordo com a equação estimada, verificou-se uma explicação da massa de dados de $88 \%$ (Tabela 2).

Entre as densidades de 41 e 79 camarões por $\mathrm{m}^{2}$, verificou-se que a produção foi crescente. Esses resultados corroboram os trabalhos de Maguire \& Leedow (1983) e Wyban et al. (1987), ao relacionarem a densidade de estocagem com o crescimento individual dos camarões e com a competição por alimento. Ao comparar os dados de produção de 2004 com 2003, observou-se um crescimento de $16,89 \%$ na produção da fazenda. Ao confrontar esse crescimento com a produção nacional do camarão cultivado, registrada para o mesmo período, uma queda de $15,8 \%$, verificou-se que a fazenda foi muito eficiente na sua linha de produção.

Ao relacionar as variáveis de manejo com a produtividade, apenas nove variáveis foram significativas para sua predição (Equação 2):
Prodt $=(119,63-1,28 \mathrm{NC}+14,71$ Aera $-0,42 \mathrm{TP}+$ $0,86 \mathrm{SAL}-2,25 \mathrm{Temp}_{\mathrm{F}}-0,000036 \mathrm{PI}+2,07 \mathrm{DE}-0,54 \mathrm{TC}$ $+0,00096 \mathrm{QRO})^{2}$, em que: Prodt é a produtividade (t); NC é o número de ciclos; Aera é a potência da aeração (HP); TP é o tempo de preparação; SAL é a salinidade $\left(\mathrm{g} \mathrm{L}^{-1}\right)$; Temp $\mathrm{F}_{\mathrm{F}}$ é a temperatura de fundo $\left({ }^{\circ} \mathrm{C}\right)$; PI é a população inicial; DE é a densidade de estocagem (camarão por $\mathrm{m}^{2}$ ); TC é o tempo de cultivo (dias); QRO é a quantidade de ração ofertada $(\mathrm{kg})$.

Ao utilizar valores máximos para as variáveis Aera, SAL, DE e QRO, a produtividade é otimizada, ao passo que com valores máximos de NC, TP, Temp ${ }_{\mathrm{F}}$, PI e TC (Tabela 1) ela é minimizada. As variáveis selecionadas na equação explicaram $80 \%$ da variabilidade da resposta.

As variáveis $\mathrm{Temp}_{\mathrm{F}}$ e SAL foram selecionadas para predizer tanto a produção quanto a produtividade. A SAL foi diretamente proporcional, enquanto que $\mathrm{T} \mathrm{Temp}_{\mathrm{F}}$ foi inversamente proporcional. Portanto, sugere-se a manutenção da salinidade em seus valores máximos e a temperatura no mínimo, para que se tenha um alto índice de produção e produtividade. Observou-se que a salinidade variou entre $23 \mathrm{e} 39 \mathrm{~g} \mathrm{~L}^{-1}$, e a temperatura no fundo do viveiro entre 27,09 e $31,07^{\circ} \mathrm{C}$, valores dentro dos padrões recomendados por Boyd (1997). Marques et al. (1999) ao testar o efeito de alta salinidade no cultivo do Litopenaeus vannamei, em laboratório, verificou que salinidade de $60 \mathrm{~g} \mathrm{~L}^{-1}$, quando associada com outros fatores adversos da água do viveiro, pode influenciar negativamente na produtividade, e que os melhores resultados foram obtidos nas salinidades de 30 e $40 \mathrm{~g} \mathrm{~L}^{-1}$. Chen \& Lin (1994) afirmaram que com a salinidade do viveiro próxima ao ponto isosmótico da hemolinfa obtém-se uma melhor produção. De acordo com Castille Junior \& Lawrence (1981) e Chaves (1989), o ponto isosmótico de juvenis de Litopenaeus vannamei está entre 24,7 e $26 \mathrm{~g} \mathrm{~L}^{-1}$.

Como a variável estação do ano (verão ou inverno) não foi selecionada nos modelos de produção e produtividade, pode-se afirmar que suas médias não foram influenciadas significativamente no período

Tabela 2. Variáveis selecionadas para os modelos de predição de produção do camarão marinho Litopenaeus vannamei(1).

\begin{tabular}{|c|c|c|c|}
\hline Variável resposta & Variáveis independentes selecionadas & $\mathrm{R}^{2}$ & $\lambda$ \\
\hline Prod & NC, AV, SAL, Temp $F$, PI, DE, QRO & 0,88 & 0,5 \\
\hline Prodt & NC, Aera, TP, SAL, Temp ${ }_{F}$, PI, DE, TC, QRO & 0,80 & 0,5 \\
\hline $\mathrm{PF}$ & $\mathrm{pH}, \mathrm{TP}, \mathrm{Temp}_{\mathrm{F}}, \mathrm{TC}, \mathrm{QRO}$, Aera, LFPL $_{\mathrm{B}}, \mathrm{LFPL}_{\mathrm{E}}$ & 0,77 & $-0,5$ \\
\hline$\underline{\text { Sob }}$ & $\mathrm{Oxi}_{\mathrm{S}}$, Temp $_{\mathrm{F}}$, PI, Transp, TC, QRO, Aera, LFPL $\mathrm{A}, \mathrm{LFPL}_{\mathrm{D}}, \mathrm{EA}_{\mathrm{V}}$ & 0,63 & 3 \\
\hline
\end{tabular}

(1)Aera: aeração; AV: área do viveiro; DE: densidade de estocagem; EA : estação do ano verão; LFPL: laboratório fornecedor de pós-larva; NC: número de ciclos; Oxi : oxigênio de superfície; PF: peso final; pH: pH; PI: população inicial; Prod: produção; Prodt: produtividade; QRO: quantidade de

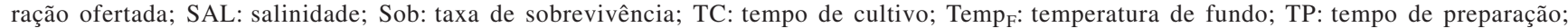
Transp: transparência da água. 
analisado. Esses resultados estão de acordo com o fato de que no Brasil, na maioria dos estados, é possível produzir camarões durante todo o ano, em razão da pouca variação climática. Ressalta-se que na região nordestina é possível se obter até três ciclos de produção por ano. A produtividade média na fazenda, no ano de 2004 , foi de $17,76 \mathrm{~kg} \mathrm{ha}^{-1}$ por dia. Comparando-se este resultado com a média nacional desse período, que foi de $12,53 \mathrm{~kg} \mathrm{ha}^{-1}$ por dia, verificou-se uma superioridade de 41,74\% em relação à média nacional.

Ao se relacionar o peso final dos camarões com os dados de manejo e dos parâmetros físicos e químicos da água dos viveiros, pode-se modelar matematicamente $\mathrm{o}$ peso de acordo com a Equação 3:

$\mathrm{PF}=\left(0,75-0,0075 \mathrm{pH}+0,0003 \mathrm{TP}-0,0118 \mathrm{Temp}_{\mathrm{F}}-\right.$ $0,0006 \mathrm{TC}-0,0000006 \mathrm{QRO}+0,0022$ Aera $+0,0093 \mathrm{LFPL}_{\mathrm{B}}$ $\left.+0,0096 \mathrm{LFPL}_{\mathrm{E}}\right)^{-2}$, em que: PF é o peso final $(\mathrm{g}) ; \mathrm{pH}$ é o pH da água do viveiro; TP é o tempo de preparação (dias); Temp $\mathrm{F}_{\mathrm{F}}$ é a temperatura de fundo $\left({ }^{\circ} \mathrm{C}\right)$; TC é o tempo de cultivo (dias); QRO é a quantidade de ração ofertada $(\mathrm{kg})$; Aera é a potência da aeração (HP); LFPL $_{B}$ e LFPL ${ }_{E}$ são os laboratórios B e E fornecedores de pós-larva, respectivamente.

Ao se ajustarem as variáveis $\mathrm{pH}, \mathrm{Temp}_{\mathrm{F}}, \mathrm{TC}$ e QRO em seus valores máximos, obtém-se a maximização do peso dos camarões; ao se utilizarem os valores máximos para as variáveis TP e Aera com pós-larvas dos laboratórios $\mathrm{B}$ ou $\mathrm{E}$, minimiza-se a variável-resposta peso final. Ao se relacionar o peso final do camarão cultivado Litopenaeus vannamei com as variáveis de manejo, sem se utilizarem as variáveis físicas e químicas da água, Ximenes (2005) obteve um índice determinístico inferior a $10 \%$. Ao se compararem os resultados de Ximenes com os obtidos no presente trabalho, $\left(\mathrm{R}^{2}=0,77\right)$ para essa relação (Tabela 2$)$, constatou-se um aumento significativo na explicação dos modelos, fato que se pode associar a inclusão das variáveis físicas e químicas da água, nos referidos modelos. O tempo de cultivo maximizou o peso final do camarão, o que corrobora o trabalho de Pereira (2001).

As variáveis físicas e químicas selecionadas na equação do peso final foram a temperatura no fundo e o $\mathrm{pH}$ da água, respectivamente, e ambas maximizaram a resposta. A variação do $\mathrm{pH}$, durante o período de estudo, foi de 6,1 a 8,92, enquanto a variação da temperatura do fundo do viveiro foi de 27,09 a $31,07^{\circ} \mathrm{C}$, que se encontram dentro dos padrões recomendados por Boyd (1997). De acordo com Lestler \& Pante (1991), a temperatura é considerada o principal parâmetro físico responsável pelo consumo de alimentos e pelo crescimento do camarão.
A taxa de sobrevivência do camarão pode ser sumarizada pela seguinte Equação 4:

$\mathrm{Sob}=\left(7238778,44-105981,98 \mathrm{Oxi}_{\mathrm{S}}-165505,09 \mathrm{Temp}_{\mathrm{F}}-\right.$ $0,33 \mathrm{PI}+5381,61$ Transp $-9948,44 \mathrm{TC}+13,05 \mathrm{QRO}+$ 13864,28Aera - 129679,76LFPL $-107991,53 \mathrm{LFPL}_{\mathrm{D}}+$ $\left.93152,48 \mathrm{EA}_{\mathrm{V}}\right)^{1 / 3}$,

em que: Sob é a taxa de sobrevivência (\%); Oxis é o oxigênio de superfície $\left(\mathrm{mg} \mathrm{L}^{-1}\right), \mathrm{Temp}_{\mathrm{F}}$ é a temperatura de fundo $\left({ }^{\circ} \mathrm{C}\right)$; PI é a população inicial de camarões; Transp é a transparência da água $(\mathrm{cm})$; TC é o tempo de cultivo; QRO é a quantidade de ração ofertada; Aera é a potência da aeração (HP); $\mathrm{LFPL}_{\mathrm{A}}$ e $\mathrm{LFPL}_{\mathrm{D}}$ são os laboratórios A e D fornecedores de póslarva, respectivamente; $E A_{V}$ é a estação de verão.

Com base na equação da sobrevivência, verificou-se que as variáveis Transp, QRO e EA $\mathrm{V}_{\mathrm{V}}$ maximizaram a sobrevivência dos camarões, enquanto Oxis, $\mathrm{Temp}_{\mathrm{F}}$, PI, TC, LFPL $_{A}$ e LFPL $L_{D}$ a minimizaram. O coeficiente de determinação para o referido modelo foi de $63 \%$. Apesar de o coeficiente ser moderadamente baixo em relação às demais variáveis respostas, verificou-se, também, um aumento significativo dessa estatística com a incorporação dos variáveis físicas e químicas, quando comparado com o obtido por Ximenes (2005), que foi de 0,14 .

A taxa de sobrevivência média, observada em relação ao período estudado, foi de $93,44 \%$ à densidade média de estocagem de 63 indivíduos por metro quadrado, com a duração do cultivo variando de 93 a 170 dias. De acordo com a classificação de Nunes \& Martins (2002), os resultados de sobrevivência podem ser considerados excepcionais, em decorrência de terem sido superiores a 90\%. Segundo Mendes (1992), a sobrevivência de camarões em cultivos é influenciada por vários fatores, entre eles, a densidade de estocagem, os parâmetros físicos e químicos da água, a deficiência alimentar, a ação dos predadores. Almeida et al. (1999) encontraram taxa de sobrevivência final de 67,5, 82,5 e $70 \%$ para as densidades de 10, 20 e 30 indivíduos por metro quadrado de Litopenaeus vannamei, respectivamente. Seabra (2004) cultivou camarão marinho Litopenaeus vannamei na densidade de 21 indivíduos por metro quadrado, obteve sobrevivência média de $80 \%$. Pereira (2001) observou que cultivos com um tempo de duração maior apresentaram taxa de sobrevivência menor.

Ao se representar graficamente a taxa de sobrevivência em função do tempo de cultivo, com base na equação 4 (Figura 1), com os valores médios das variá- 


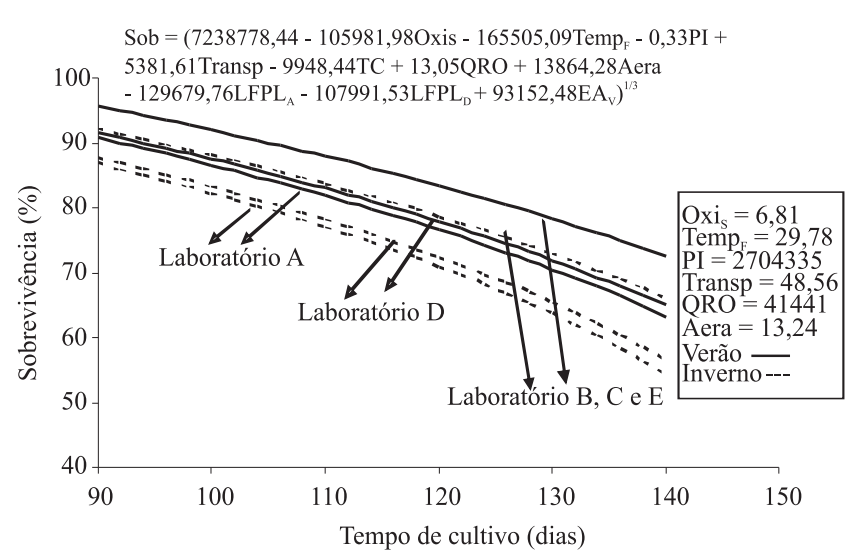

Figura 1. Curva de sobrevivência do camarão Litopenaeus vannamei submetido a diferentes estratégias de manejo.

veis TR, RA, AE, OS, TF, PI (Tabela 1), verificou-se que a sobrevivência dos camarões foi influenciada, inversamente, pelo tempo de cultivo. Observou-se, também, que as pós-larvas dos laboratórios $\mathrm{B}, \mathrm{C}$ e $\mathrm{E}$ proporcionaram os melhores resultados em relação à taxa de sobrevivência.

Predições baseadas nos modelos encontrados, para as variáveis-respostas em estudo, não podem ser feitas com valores acima ou abaixo dos limites apresentados na Tabela 1. Segundo Downing \& Clark (1998), predições feitas em situações em que os valores estão fora dos intervalos observados (extrapolações) podem acarretar erros de estimação.

\section{Conclusões}

1. Podem-se relacionar matematicamente as variáveis produção, produtividade, taxa de sobrevivência e peso final do Litopenaeus vannamei com as de manejo.

2. As variáveis de manejo que mais influenciam nos resultados são tempo de cultivo, quantidade de ração ofertada e densidade de estocagem.

3. A origem das pós-larvas exerce influência significativa nos resultados do cultivo.

\section{Agradecimentos}

À Finep, pelo apoio à Rede de Carcinicultura para Pesquisadores (Recarcine), em especial ao projeto Gerenciamento de Banco de Dados.

\section{Referências}

ALMEIDA, S.A.A.; CÉSAR, J.R.O.; IGARASHI, M.A.; BEZERRA, F.J.S.; CARVALHO, M.C. Estudo preliminar do cultivo de Penaeus vannamei (Boone, 1931) em tanques com diferentes densidades de estocagem. In: CONGRESSO BRASILEIRO DE ENGENHARIA DE PESCA, 11.; CONGRESSO LATINOAMERICANO DE ENGENHARIA DE PESCA, 1., 1999, Recife. Anais. Recife: Associações dos Engenheiros de Pesca de Pernambuco, 1999. v.2, p.648-653.

BOYD, C.E. Manejo do solo e da qualidade de água em viveiros para aqüicultura. Tradução: Eduardo Ono. Campinas: Associação Americana de Soja, 1997. 55p.

CASTILLE JUNIOR, F.L.; LAWRENCE, A.L. The effect of salinity on the osmotic, sodium and chloride concentrations in the hemolymph of euryhaline shrimp of the genus Penaeus. Comparative Biochemistry and Physiology A, v.68, p.75-80, 1981.

CHAVES, L. El efecto de la salinidad sobre la osmolaridad hemolinfática de los peneideos. In: SIMPÓSIO BRASILEIRO SOBRE CUlTIVO DE CAMARÃO, 3., 1989, João Pessoa. Anais. João Pessoa: MCR Aquacultura, 1989. p.541-557.

CHEN, J.C.; LIN, J.L. Osmolality and chloride concentration in the hemolymph of subadult Penaeus chinensis subjected to different salinity levels. Aquaculture, v.125, p.167-174, 1994.

CORDEIRO, G.M.; NETO, E.A.L. Modelos paramétricos. Recife: Associação Brasileira de Estatística, 2004. 246p.

DOWNING, D.; CLARK, J. Estatística aplicada. Tradução: Alfredo Alves Farias. São Paulo: Saraiva, 1998. 455p.

FAO. Fishery Information, Data and Statistics Unit. FishStat plus: universal software for fishery statistical time series. Version 2.3. Rome, 2006. Disponível em: <http://www.fao.org/fi/statist/FISOFT/ FISHPLUS.asp>. Acesso em: 6 maio 2006.

LESTLER, L.J.; PANTE, J.R. Penaeids temperature and salinity responses. In: FAST, A.W.; LESTLER, L.J. Marine shrimp culture: principles and practices. Amsterdam: Elsevier, 1991. p.515-534. (Developments in aquaculture and fisheries science, 23).

MAGUIRE, G.B.; LEEDOW, M.I. A study of the optimum stocking density and feed rate for school prawns Metapenaeus macleayi (Haswell) in some Australian brackish water farming ponds. Aquaculture, v.30, p.285-297, 1983.

MARQUES, L.C.; GESTEIRA, T.C.V.; ANDRADE, T.P.; CARVALHO, R.L.; CAVALCANTE, F.A.M.; ARAÚJO, P.H.G.; MARTINS, P.C.C.; HENNIG, O. Efeito de altas salinidades sobre o cultivo do camarão da espécie Litopenaeus vannamei (Boone, 1931) em condições de laboratório. In: CONGRESSO BRASILEIRO DE ENGENHARIA DE PESCA, 11.; CONGRESSO LATINOAMERICANO DE ENGENHARIA DE PESCA, 1., 1999, Recife. 
Anais. Recife: Associações dos Engenheiros de Pesca de Pernambuco, 1999. v.2, p.581-588.

MENDES, P.P. Crescimento e sobrevivência do camarão, Macrobrachium rosenbergii (De Man, 1879), cultivado em diferentes colunas de água. 1992. 96p. Tese (Doutorado) Universidade Federal de São Carlos, São Carlos.

MENDES, P.P. Estatística aplicada à aquiicultura. Recife: Bagaço, 1999. 265p.

MONTGOMERY, D.C.; PECK, E.A. Introduction to linear regression analysis. New York: Wiley, 1982. 504p.

NUNES, A.J.P. Guia Purina: fundamentos da engorda de camarões marinhos. 2.ed. São Lourenço da Mata: Purina do Brasil, 2004. 42p. Disponível em: <www.purinabrasil.com.br>. Acesso em: 19 mar. 2004.

NUNES, A.J.P.; MARTINS, P.C. Avaliando o estado de saúde de camarões marinhos na engorda. Panorama de Aqüicultura, v.12, p.23-33, 2002.

PEREIRA, E.M.A. Análise dos parâmetros de crescimento do camarão de água doce Macrobachium rosenbergii (De Man, 1879), cultivado em tanques rede. 2001. 94p. Dissertação (Mestrado) Universidade Federal Rural de Pernambuco, Recife.

RODRIGUES, J. Carcinicultura marinha - desempenho em 2004. Revista da Associação Brasileira de Criadores de Camarão, v.7, p.38-44, 2005.

SEABRA, M.E. Cultivo de camarão marinho Litopenaeus vannamei em sistema multifásico. 2004. Dissertação (Mestrado) - Universidade Federal Rural de Pernambuco, Recife.

SOKAL, R.R.; ROHLF, F.J. Biometry: the principles and practice of statistics in biological research. New York: W. H. Freeman and Company, 1995. 850p.

WYBAN, J.A.; LEE, C.S.; SATO, V.T.; SWEENEY, J.N.; RICHARDS JUNIOR, W.K. Effect of stocking density on shrimp growth rates in manure-fertilized ponds. Aquaculture, v.61, p.2332, 1987.

XIMENES, N.P. Aplicação de modelos lineares na estimação dos parâmetros do cultivo do camarão marinho Litopenaeus vannamei (Boone, 1931). 2005. 64p. Dissertação (Mestrado) Universidade Federal Rural de Pernambuco, Recife.

Recebido em 2 de junho de 2006 e aprovado em 28 de novembro de 2006 